\title{
Elaboración y validación de estrategias para la enseñanza y el aprendizaje de español como lengua materna
}

\author{
Development and validation of strategies for teaching and \\ learning Spanish as a mother tongue
}

Sandoval, Armando José; Escobar Soriano, Álvaro

\author{
Armando José Sandoval \\ armando.sandoval@unan.edu.ni \\ UNAN-Managua, Nicaragua \\ iD Álvaro Escobar Soriano \\ aescobar@unan.edu.ni \\ UNAN-Managua, Nicaragua
}

Revista Torreón Universitario

Universidad Nacional Autónoma de Nicaragua-Managua,

Nicaragua

ISSN: $2410-5708$

ISSN-e: 2313-7215

Periodicidad: Cuatrimestral

vol. 10 , núm. 28,2021

revis.torreon.faremc@unan.edu.ni

Recepción: 01 Octubre 2020

Aprobación: 22 Abril 2021

URL: http://portal.amelica.org/ameli/
jatsRepo/387/3872112002/index.html

DOI: https://doi.org/10.5377/rtu.v10i28.11523

El autor o los autores de los artículos, ensayos o investigaciones conceden a la Universidad Nacional Autónoma de Nicaragua, Managua (UNAN-Managua) los derechos de edición (copyright) del trabajo enviado, por consiguiente la Universidad cuenta con el derecho exclusivo para publicar el artículo durante el periodo completo de los derechos de autor.

Esta obra está bajo una Licencia Creative Commons AtribuciónNoComercial-SinDerivar 4.0 Internacional.
Resumen: En el presente artículo se abordan los detalles sobre el proceso de investigación del proyecto titulado: Elaboración y validación de estrategias para la enseñanza y el aprendizaje de español como lengua materna, trabajo que se realizó con estudiantes de la carrera de Lengua y Literatura Hispánicas. Esto inició con el análisis de trabajos didácticos resguardado en el Centro de Documentación del departamento de español (CEDOC), se encontraron un total de 45 trabajos, divididos entre las diferentes macrohabilidades de la lengua materna. Por lo tanto, los estudiantes se dividieron en equipo para trabajar: escritura, expresión oral, comprensión lectora, gramática y literatura. Se continuó con la capacitación sobre el modelo de aprendizaje por esquema (Escobar, 2016). La etapa concluye con la utilización del modelo para reestructurar los trabajos y elaborar una nueva secuencia didáctica, de los cuales se obtuvieron cinco trabajos transformados con base en el modelo. Con el proceso se está fortaleciendo la competencia didáctica y de investigación en los estudiantes.

Palabras clave: Didáctica, esquema, investigación, Lengua materna, subesquema.

Abstract: This article addresses the details during the first phase of the research process of the project entitled Elaboration and validation of strategies for the teaching and learning of Spanish as a mother tongue, work that was carried out with students of the Hispanic Language and Literature career. This began with the analysis of didactic works kept in the Documentation Center of the Spanish department (CEDOC), a total of 45 works were found, divided between the different macro-skills of the mother tongue. Therefore, the students were divided into teams to work: writing, speaking, reading comprehension, grammar and literature. Training on the schema learning model continued (Escobar, 2016). The stage concludes with the use of the model to restructure the works and develop a new didactic sequence, of which five transformed works were obtained based on the model. With the process, the teaching and research competence of the students is being strengthened.

Keywords: Didactics, scheme, research, Mother tongue, subschema. 


\section{INTRODUCCIÓN}

En el presente artículo se abordan los detalles del proceso de investigación del proyecto titulado Elaboración y validación de estrategias para la enseñanza y el aprendizaje de español como lengua materna, trabajo que se realizó con estudiantes de la carrera de Lengua y Literatura Hispánicas, porque es de conocimiento que los estudiantes de secundaria tienen dificultad para aprender. Aunque los docentes de secundaria, con el acompañamiento del Ministerio de Educación, han recibido intensos procesos de capacitación y actualización. No obstante, estos requieren de referentes metodológicos específicos que les permitan hacer frente a situaciones de aprendizaje que no son consideradas en los talleres. Por eso, el principal objetivo, de este proyecto, es elaborar recursos para la enseñanza y el aprendizaje en lengua materna. Este se desarrolló en dos fases: la creación de plantillas para la estructuración de estrategias de enseñanza y aprendizaje y la reestructuración de las estrategias encontradas en los trabajos de investigación en didáctica de la lengua materna.

\section{MÉTodos}

Antes de hablar del modelo de aprendizaje por esquema es necesario abordar la teoría base del modelo. Esta se encuentra en la teoría esquemática, el esquema "Es la organización invariante de la conducta para una clase de situaciones dada. También es una totalidad dinámica organizadora de la acción del sujeto para una clase de situaciones específicas" (Escobar, 2016 p. 183). En él se concentra las pautas para el análisis de los aspectos que no varían en las acciones de las personas, que podrían ser observados, desarrolladas y finalmente evaluadas son, la conducta y la acción. Es decir, que el esquema no solo está cargado de la conducta, sino de algo más activo, la acción porque la actividad humana está regida por conceptos y estos se movilizan a través de la acción. Entonces, es esta teoría que sustenta el modelo a que se utilizó en la elaboración de la secuencia didáctica. Además, las funciones mencionadas ocurren en la mente de la persona e intervienen en el aprendizaje del estudiante, por lo cual, es necesario darle la atención que amerita.

Por otra parte, un esquema está conformado por subesquemas, que contienen aspectos específicos, pero que se complementan. En ese sentido, los subesquemas se clasifican en: subesquema conceptual, está relacionado con el significado de las palabras; subesquema lingüistico se relaciona con la formación de las palabras e ideas que se utilizan en los diferentes momentos; en cambio el subesquema sociocultural se relaciona con la ideología de cada persona y esta es formada por el contacto con la familia y sociedad; finalmente, el subesquema técnico-procedimental toma toda la información contenida y desarrollada en los otros subesquemas y las vuelve acción para resolver las actividades propuestas en el escenarios que se encuentre. Por lo tanto, un subesquema no es mayor al otro, ni funciona de forma independiente, ellos están unidos, porque así funcionan en el cerebro de cada persona. Y bajo esa teoría se forma el modelo:

\section{El modelo de aprendizaje por esquema}

Es a partir de la teoría subesquemática que se propone el modelo de aprendizaje por esquema, este cuenta con las siguientes concepciones de aprendizaje asumidas: constructivista humanista, sociocultural y centrada en la comunidad, centrado en el lenguaje, con planteamiento lúdico, mediación con ayuda de la tecnología y centrado en la experiencia y vivencias (Escobar, 2016).

De forma puntual el modelo cuenta con momentos de aprendizaje:

- Evolución conceptual-factual (apropiación de invariantes conceptuales)

- Apropiación y desarrollo metalingüístico (explicitación de las invariantes conceptuales)

- Apropiación técnicas-procedimentales (atención y detección de modelos y patrones de análisis, reconstrucción de invariantes procedimentales) 
- Aplicación procedimental (resolución de problemas): (Escobar, 2016, p.692-697)

Los momentos de aprendizaje del modelo guían las actividades y estas responden directamente al proceso de aprendizaje. En este sentido, el modelo busca responder a las necesidades de aprendizaje, porque se trabaja desde la exploración de conocimientos, hasta la puesta en práctica de la teoría estudiada. Es así, que se contribuye a alcanzar las competencias del grado en que se aplica la estrategia. Por eso, se puede decir que desde el enfoque por competencia es busca poner en práctica los conocimientos, eso es la misma búsqueda del modelo de aprendizaje por esquema.

El trabajo se realiza con una visión social que integra los diferentes puntos del quehacer educativo, teoría y práctica (González, 2003), por eso la presente investigación es bajo el paradigma sociocrítico. Por otra parte, es el enfoque cualitativo permitió hacer la descripción del proceso (Hernández, Fernández y Baptista, 2006) y relacionarlo con los elementos que intervienen en la construcción de una estrategia didáctica en lengua materna. Además, el tipo de investigación es investigación-acción, porque el docente investiga en su propia práctica y el estudiantado pasa a ser parte del proceso y a proponer de forma conjunta soluciones (Restrepo 2002). Por otra parte, el proceso apunta a una innovación pedagógica, porque se operativiza el modelo de aprendizaje por esquema (Escobar, 2016) en estudiantes de la carrera, y estos a su vez elaborarán una estrategia didáctica para aplicarla en las aulas de secundaria. Por lo tanto, esto permite realizar ajustes en el proceso de formación de los futuros docentes e innovar estrategias para aplicarse en la solución de problemáticas reales existentes en las aulas de secundaria y además contrarrestar las prácticas de la enseñanza tradicional. Por consiguiente, los estudiantes de la carrera de lengua y Literatura Hispánicas, a quieren conciencia sobre la competencia investigativa y de la didáctica en lengua materna como herramienta para solucionar problemas educativos.

La población para este estudio fueron 30 estudiantes de cuarto año de la carrera de Lengua y Literatura Hispánicas del primer semestre de 2018. Estos cursaban la asignatura de Didáctica de la lengua y literatura en la modalidad por encuentro (sabatino) en la Universidad Nacional Autónoma de Nicaragua, Managua (UNAN-Managua). Fueron seleccionados porque ellos serán docentes de secundaria en el futuro. Por lo tanto, su participación potenciaría sus capacidades, formándoles conciencia para resolver problemas reales con la aplicación de una estrategia didáctica y además de participar en un proceso de investigación. Sin embargo, el proyecto terminó con 14 estudiantes, porque unos decidieron botar la clase por parecerles difícil la investigación y otros no regresaron a clase por la situación pol que se vivió en el país en el 2018.

Se aplicaron técnicas como entrevista, observación, encuesta, estas acompañadas de sus instrumentos, lista de cotejo, guía de observación, guía de preguntas y el trabajo final de los estudiantes (la secuencia didáctica). El análisis se realiza mediante estudio de caso. En ese sentido, cada participante y sus instrumentos son un caso para ser analizado. El mayor peso lo tendrá la secuencia elaborada y con ella es producto del dominio del modelo de aprendizaje por esquema.

La metodología de análisis se desarrolló en dos fases: a) creación de plantilla para la estructuración de estrategias de enseñanza y aprendizaje, aplicando el modelo de esquemas de aprendizaje, y b) reestructuración de las estrategias de aprendizaje, encontrados en los trabajos de investigación en didáctica de la lengua materna, realizados en el Departamento de Español, y creación de nuevos recursos de enseñanza y aprendizaje de las macrohabilidades del lenguaje, utilizando la plantilla creada.

La primera fase comprenderá dos momentos: a) la elaboración de la plantilla de trabajo para la elaboración de estrategias de enseñanza y aprendizaje basada en el modelo esquemas de aprendizaje y, b) la capacitación a los estudiantes sobre el modelo de aprendizaje basado en esquemas. Esta se realizará mediante la metodología de taller, con la que el equipo aplicará la plantilla elaborada para reestructurar recursos de enseñanza y aprendizaje que se encuentren en los informes de investigación y elaborar nuevos recursos. 


\section{Resultados}

En esta parte se involucraron catorce estudiantes, quienes participaron en la capacitación sobre la apropiación del modelo de aprendizaje por esquema mediante la metodología de taller, con ello se comprendió la estructura de la plantilla.

A continuación se presenta y se analiza la plantilla:

\section{Secuencia Didáctica}

Secuencia Didáctica basada en el Modelo de aprendizaje por Esquemas

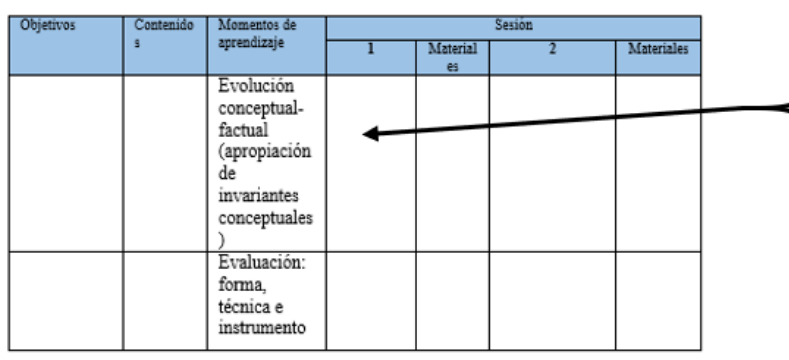

En este momento se redactan objetivos: conceptual, procedimental y actitudinal. Los discentes realizan acciones relacionadas con:

Exploración de los conceptos y teoremas aprendidos.

Exploración de la aplicación de las invariantes operatorias conceptuales

Explicitación de las invariantes procedimentales que acompañan a las invariantes conceptuales.

Detectar los errores en las invariantes operatorias.

Plantilla propia con base en el modelo de aprendizaje por esquema (Escobar, 2016)

Es decir, que en este primer momento del modelo de aprendizaje por esquema el docente explorará los conocimientos conceptuales y procedimentales almacenados en el cerebro con relación al gran esquema que se esté trabajando, además como el modelo por esquema es con base en teoría de las ciencias cognitivas se provee el conocimiento para detectar las fallas y dar solución a las mismas. Por lo tanto, se podrá incidir de forma directa en la superación del problema

\begin{tabular}{|l|l|l|l|l|}
\hline Objetivos & Contenidos & $\begin{array}{l}\text { Momentos de } \\
\text { aprendizaje }\end{array}$ & \multicolumn{2}{|c|}{ Sesión } \\
\cline { 3 - 5 } & & $\begin{array}{l}\text { Apropiación y } \\
\text { desarnollo } \\
\text { metalinguistico } \\
\text { (explicitación de las } \\
\text { invarantes } \\
\text { conceptuales) }\end{array}$ & & \\
\cline { 3 - 5 } & & $\begin{array}{l}\text { Evaluación: forma, } \\
\text { técnica instrumento }\end{array}$ & & \\
\hline & & & \\
& & & & \\
\hline
\end{tabular}

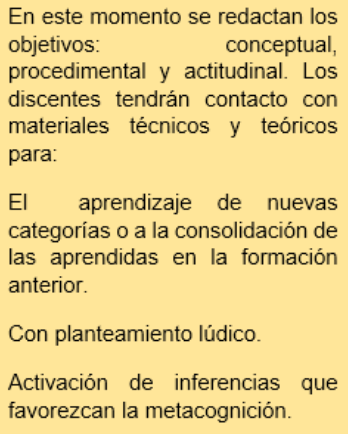

Con el segundo momento del modelo se orienta trabajar con la nueva formación teórica relacionada al contenido que representa el gran esquema que se persigue desarrollar. Este momento está relacionado con los subesquema conceptual-factual, sociocultural, lingüístico, porque los subesquemas no funcionan separados, por el contrario funcionan de forma sistémica. Es decir, la información se comparte con todos los subesquema para reforzar el conocimiento conceptual y este se evidencie en lo procedimental. 


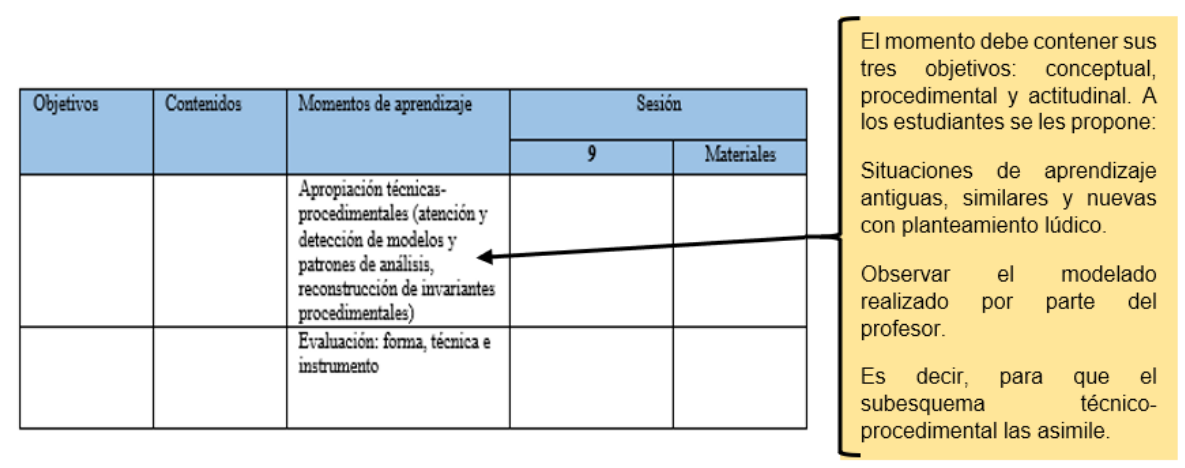

El modelo en este momento le propone al docente como guía del proceso realice modelado de técnicasprocedimentales que le sirvan al estudiantado, para la realización de su trabajo de forma independiente. La modelación tiene el objetivo que el estudiantado asimile patrones procedimentales que aplicará en el futuro en el trabajo independiente.

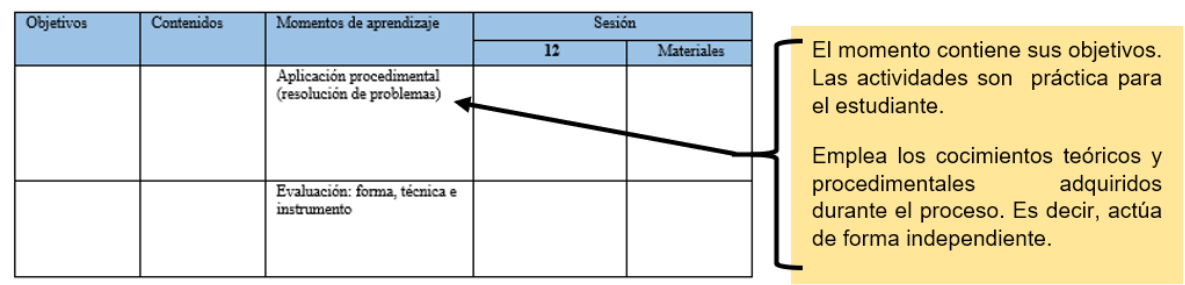

Es este momento es el punto medular del modelo, porque es donde el estudiante pondrá en práctica los conocimientos conceptuales, procedimentales y actitudinales, formados en las sesiones de cada momento. Sin embargo, se puede decir que no es el final del proceso, sino la continuidad del proceso cíclico.

Un aspecto que es necesario completar del modelo de aprendizaje por esquema es lo relacionado a la evaluación. Este momento del modelo está presente en cada uno de los otros momentos, como se muestra en la plantilla, el cual cuenta con las formas, técnicas e instrumentos para evaluar las sesiones de clase. Con este se va observando los avances de los discentes de un momento al otro.

Luego de apropiarse de la plantilla los estudiantes procedieron a la reestructuración del trabajo seleccionado con el modelo de aprendizaje por esquema. Estos corresponden a los encontrados en el CEDOC de español.

Presentación y análisis de uno de los trabajos elaborados por los estudiantes de cuarto año de la carrera de Lengua y Literatura Hispánicas de la UNAN-Managua basada en el modelo de aprendizaje: 


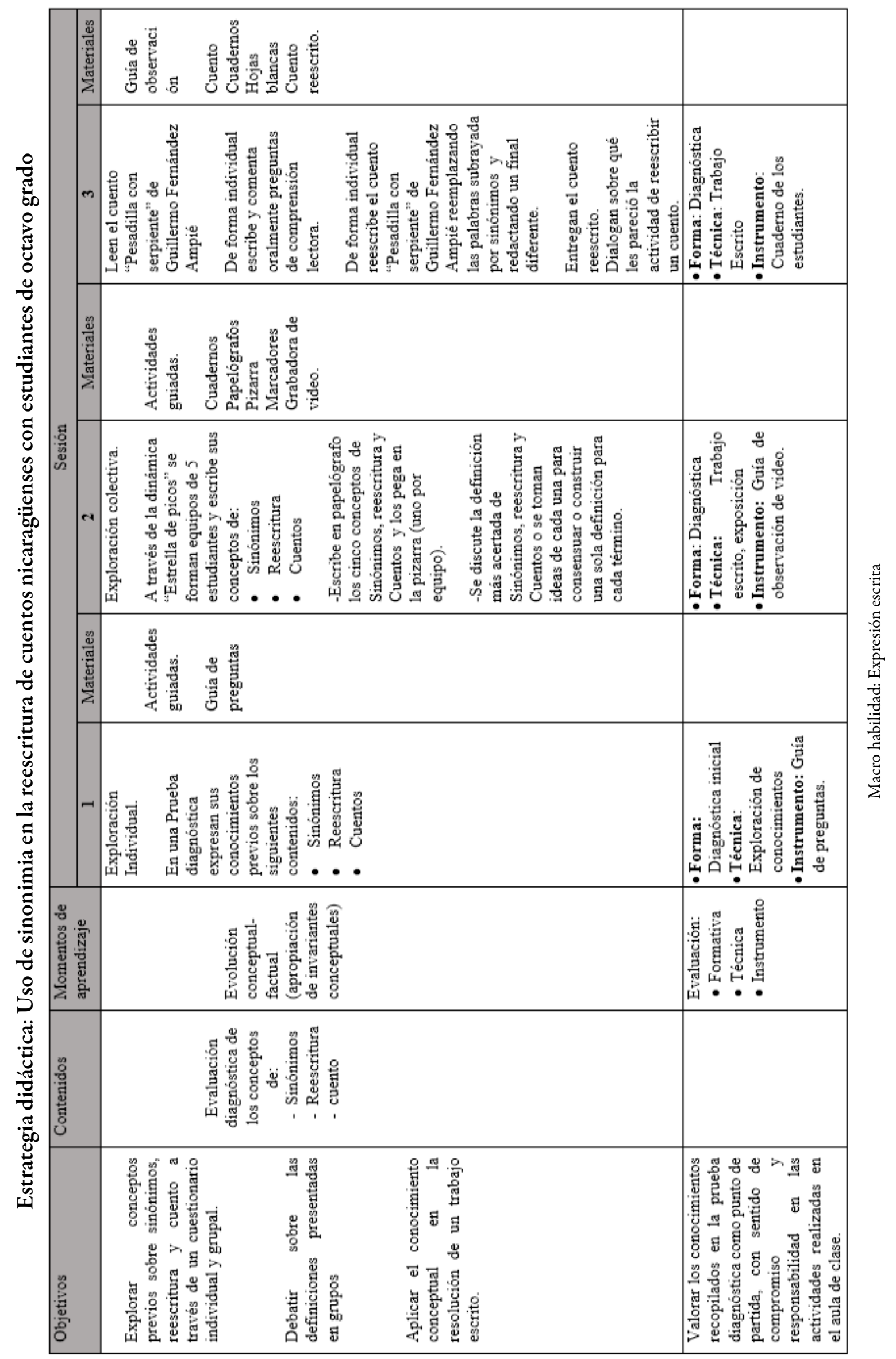




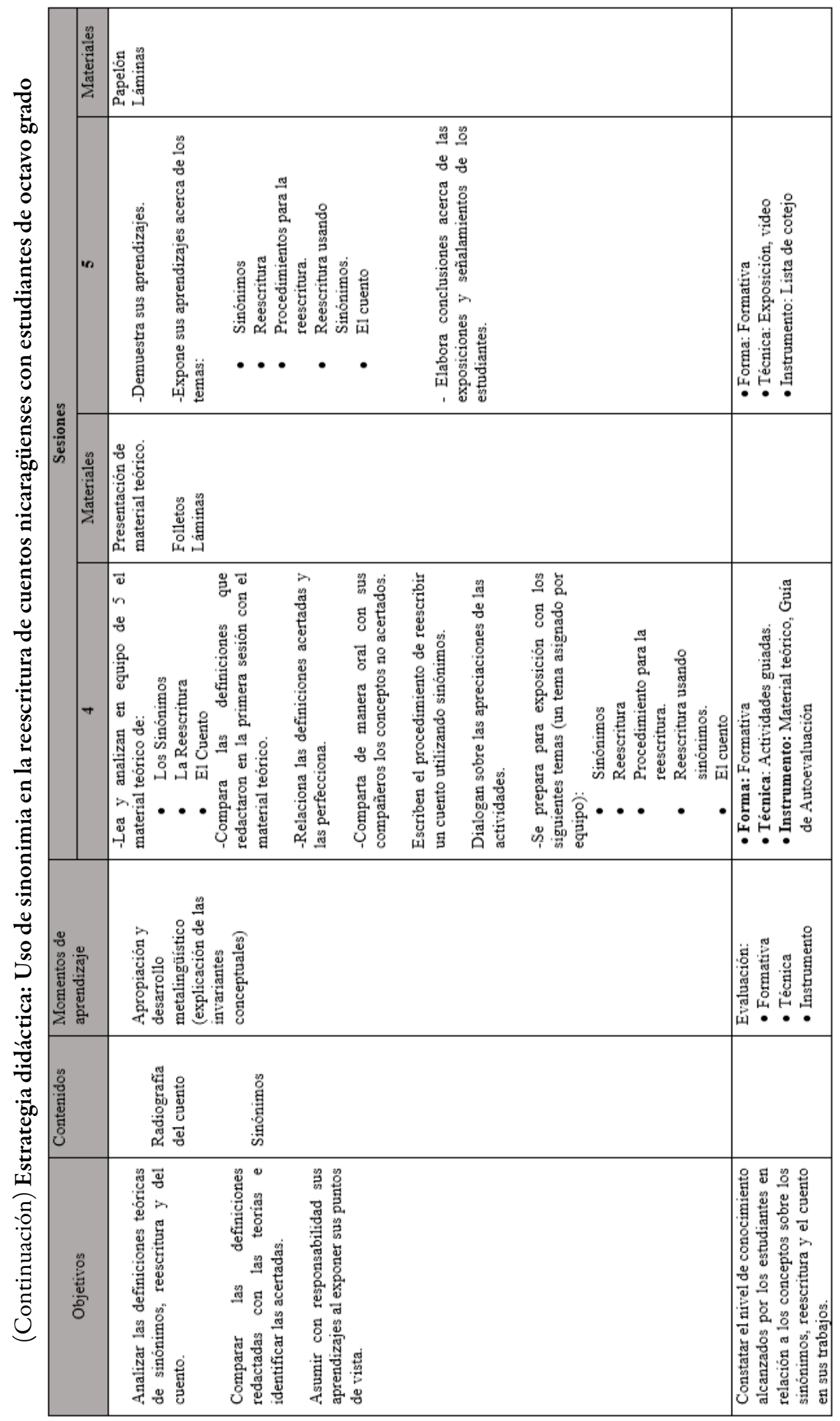




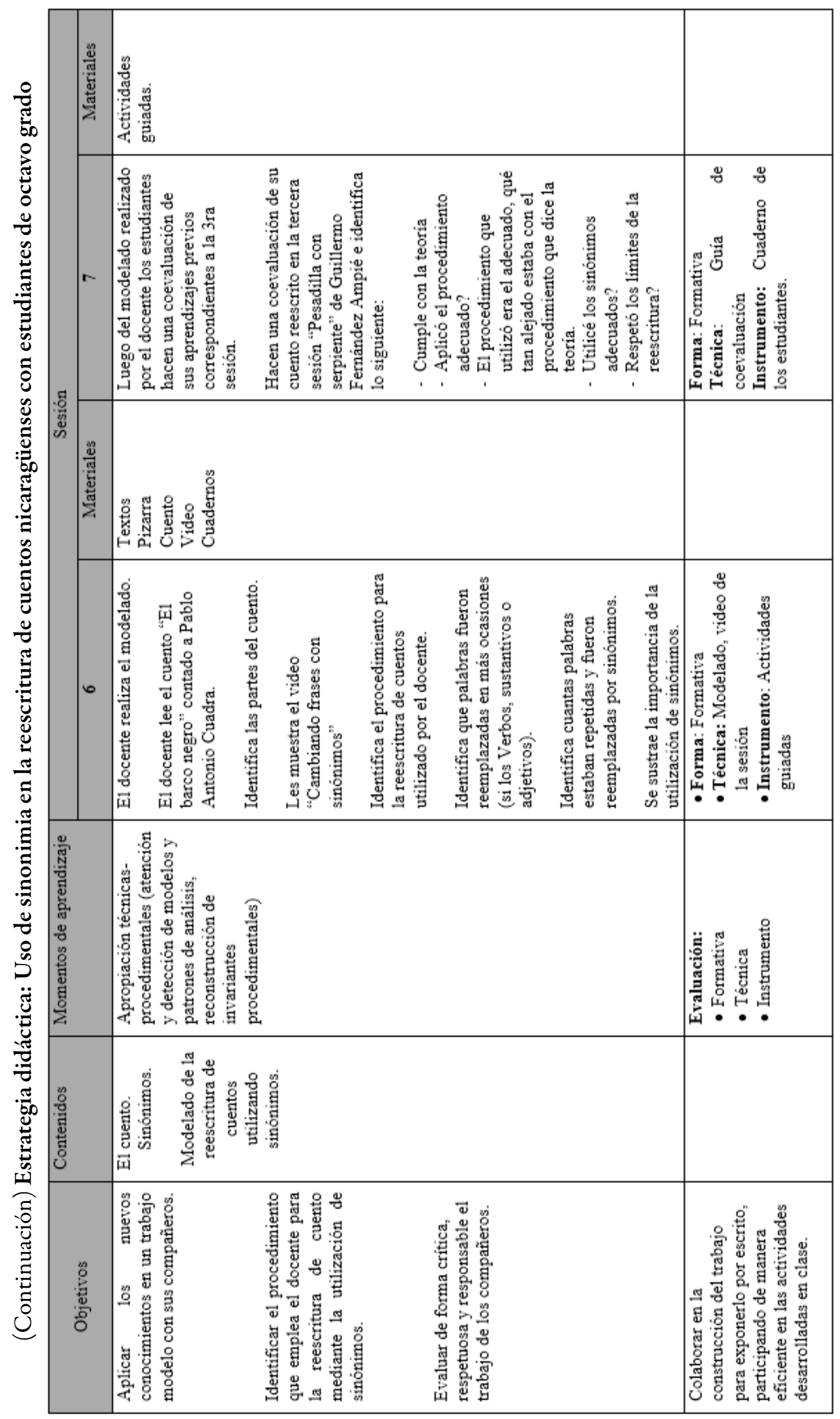




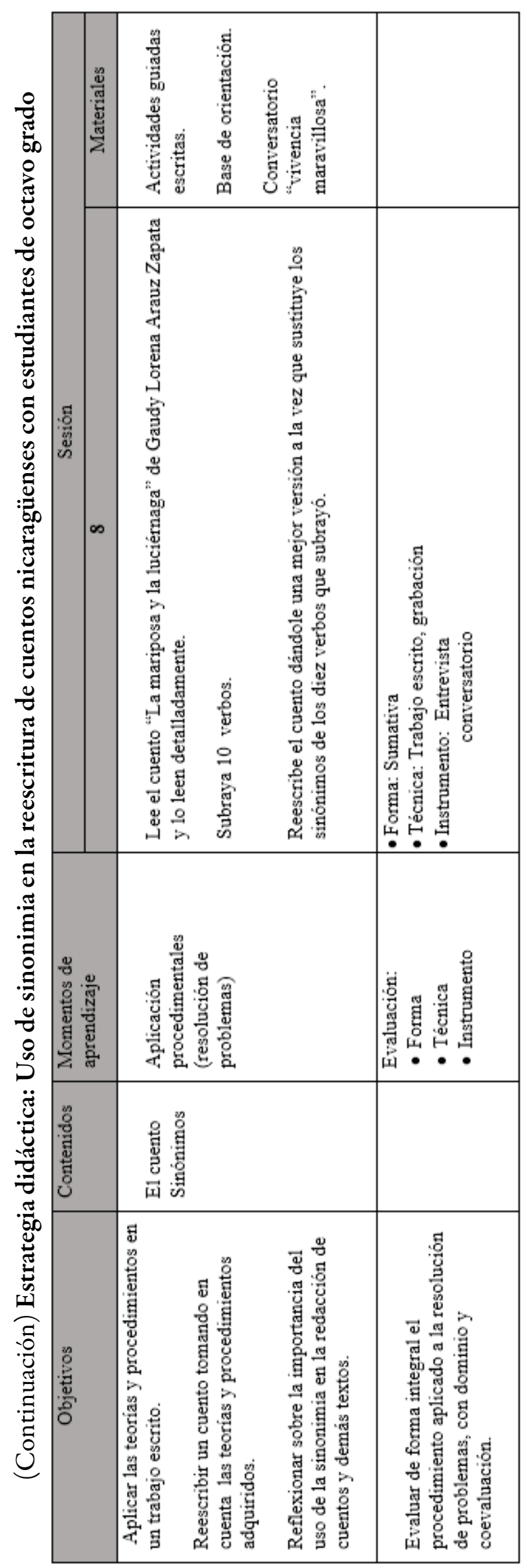


El trabajo que se presenta cumplió con las indicaciones de cada uno de los momentos del modelo de aprendizaje que se puntualizan:
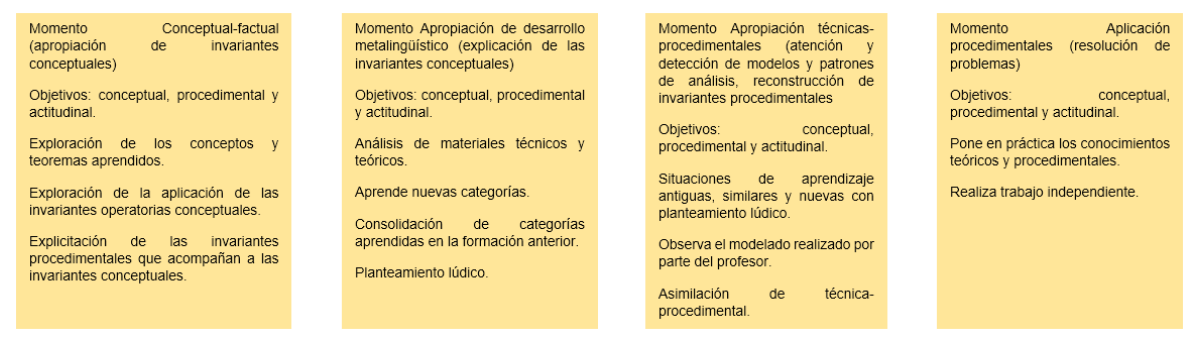

\section{CoNCLUSIÓN}

El proceso realizado permitió llegar a las siguientes conclusiones:

- El curso lo iniciaron treinta estudiantes, sin embargo catorce concluyeron el semestre, estos fueron capacitados en el uso del modelo de aprendizaje por esquemas.

- Se elaboraron siete estrategias de enseñanza aprendizaje, mediante el proceso de reestructuración de los informes de investigación del CEDOC del Departamento de Español, mediante el modelo de aprendizaje por esquema.

- El Dr. Álvaro Antonio Escobar Soriano apoyó todo el proceso de elaboración de la estrategia de enseñanza aprendizaje basada en el modelo de aprendizaje por esquema.

- La utilización de metodología participativa contribuyó en el proceso de adquisición teórica del modelo de aprendizaje por esquema.

- Se recibió la laptop por parte de la administración de la Facultad de Educación e Idiomas, esta se compró con la beca otorgada por los Fondos de Proyectos de Investigación.

- Las estrategias reestructuradas formarán parte del proyecto de investigación: Publicación de libro sobre didáctica de la lengua materna, que elabora el Dr. Álvaro Antonio Escobar Soriano.

\section{REFERENCIAS}

Escobar, A. (2016). Esquemas de aprendizaje de la gramática (Doctoral dissertation, Tesis Doctoral. Managua: UNAN-Managua).

Hernández, F., Fernández, C. \& Baptista Lucio, P. (2006). Metodología de la Investigación. Cuarta edición. Mac Graw Hill editores. México DF México, 103, 205

Morales, A. G. (2003). Los paradigmas de investigación en las ciencias sociales. Islas, (138), 125-135.

Restrepo, M. (2002). Investigación en educación. (Modulo 7). Colombia; ARFO 